Produto \& Produção, vol. 11, n. 3, p. 29-44, out. 2010

\title{
Cálculo da complexidade organizacional em dois arranjos produtivos da indústria calçadista
}

\author{
Miguel Afonso Sellitto \\ PPGEPS - UNISINOS \\ sellitto@unisinos.br \\ Marcelo Giovani Guimarães \\ UNISINOS \\ guimaraes.m@marisolrs.com.br
}

Este artigo apresenta uma metodologia quantitativa para cálculo e eventual controle da complexidade em arranjos interorganizacionais. A pesquisa propôs e testou uma metodologia para calcular a complexidade relativa de um arranjo pela quantidade de informação que as partes do arranjo devem trocar para fins de gestão. $\mathrm{O}$ teste foi realizado em dois arranjos presentes na indústria calçadista: uma cadeia de suprimentos e uma rede de ateliers de costura. Entende-se que estes tipos de arranjos organizacionais apresentam características que podem ser descritas segundo a teoria da complexidade e que seja desejável conhecer e controlar sua complexidade por meio do índice calculado. A complexidade calculada da cadeia foi de $12,67 \%$ e a da rede foi de $22,67 \%$.

Palavras-chave: Complexidade, estratégia complexa, não-linearidade, arranjos interorganizacionais, indústria calçadista.

This paper presents a quantitative methodology for calculation and eventually control of complexity in interorganizational arrays. The research proposed and tested a methodology for calculation of the relative complexity in an array by the quantity of information changed by parts, for management sake. The test was led in two arrays of the footwear industry: a supply chain and a network of industrial services. We believe that such arrays have characteristics of complex adaptive systems that can be described by complexity theory and that would be desirable to know and control their complexity by and index. The calculated relative complexity was $12,67 \%$ in the supply chain and $22,67 \%$ in the network.

Keywords: Complexity, complex strategy, non-linearity, interorganizational arrays, footwear industry.

\section{Introdução}

No atual cenário de negócios, relações lineares de causa e efeito não são mais suficientes para explicar os fenômenos resultantes das complexas interações que surgem dentro e entre organizações. Estas interações surgem principalmente em sistemas de produção que se organizam em arranjos compostos por múltiplas empresas (SELLITTO et al., 2008b), tal como ocorre em cadeias de suprimentos e em redes de cooperação empresarial.

Em arranjos empresariais, os efeitos observados que resultam da interação entre empresas são homólogos aos observados em outros campos da ciência, tais como as ciências naturais. Nestes, a ciência da complexidade busca explicar como os efeitos complexos podem surgir a partir do comportamento das partes e das relações necessárias entre elas. No estudo dos arranjos empresariais, pode-se tentar explicar o complexo comportamento das principais variáveis, observado do exterior, em raciocínio homólogo ao das ciências naturais.
Assim como cientistas naturais produziram interpretações mecanicistas da natureza, cientistas sociais produziram concepções homólogas para explicar os fenômenos observados em estruturas empresariais. Tais concepções admitem relações lineares de causa-efeito, ignoram incertezas e circularidades e admitem ser possível chegar a um estado de permanente equilíbrio entre entradas e saídas, desde que se controle o trabalho com suficiente rigor. Para tanto, seriam necessárias técnicas de controle do trabalho, tais como especificação, divisão, mensuração, otimizações locais e balanceamento nas tarefas (BAUER, 1999). Para Agostinho (2003), este controle rigoroso tem se tornado mais improvável, haja vista as interações, as emergências e as novas combinações propiciadas pelos sistemas e tecnologias de comunicação e informação e pela racionalidade limitada de decisores, observada principalmente quando os problemas de decisão incluem novas, múltiplas e intrinsecamente imbricadas alternativas de 
decisão. Para Khisty e Arslan (2005), o gerenciamento não é mais mecanicista, pois gestores têm se deparado com elementos que interagem de modo inesperado e não-linear, que influenciam e são influenciados pelo ambiente e pelo sistema de medição de desempenho.

No gerenciamento atual, não se pode mais ignorar a existência, dentro das organizações, de subsistemas organizacionais que evoluem de modo próprio, irreversível e imprevisível ao longo do tempo, gerando comportamento complexo. Pesquisadores da ciência do gerenciamento têm estudado as relações entre empresas e a evolução dos sistemas empresariais com base na ciência da complexidade, um movimento transdisciplinar que se afirmou como contraponto aos artificialismos e simplificações disciplinares observados até então nas ciências (SELLITTO et al., 2008b).

A ciência ou teoria da complexidade nasceu do esforço transdisciplinar de pesquisadores de diversos campos de conhecimento e pode ser considerada transição entre dois paradigmas de ciência. O primeiro inclui a ciência clássica e linear, que simplifica a realidade e reduz o objeto de estudo aos seus elementos constituintes para análise e comunicação dos achados de pesquisa por meio de modelos simplificados e reproduzíveis. O segundo também é chamado de pensamento complexo e inclui uma ciência não-linear que evita a simplificação e estuda o objeto no seu todo, incluindo as interações que surgem entre as partes e formam a sua complexidade (MORIN e LE MOIGNE, 2000). Para Prigogine (1996), a ciência da complexidade não descarta dados que não se ajustam ao modelo, mas os considera evolução e singularidade presentes na realidade observada.

Para Morin (2002; 2005), complexidade é o conjunto de ações, interações, retroações, determinações e acasos que formam a realidade, com traços de desordem, ambigüidades e incertezas, sustentadas por três princípios: (i) o dialógico, que promove a dualidade mantendo a unidade, ao aceitar que princípios contraditórios, tais como a ordem e a desordem, convivam em um objeto; (ii) o recursivo, que estabelece que efeitos observados são ao mesmo tempo causas dos processos que os produzem; (iii) e o hologramático, que estabelece que a parte está no todo como o todo está na parte, pois as partes possuem características do todo e o todo reflete propriedades das partes. Para Sterman (2000), complexidade é o número de componentes, variáveis ou estados que um objeto ou sistema pode assumir ou o número de combinações entre variáveis em um eventual problema de decisão sobre o objeto. Para Gell-Mann (2000), é o número de informações necessárias para descrever o sistema. Para Klir (1991), é o número de variáveis, estados, partes, relações e interações ativas no sistema. Para Schoderbek et al. (1975, apud JACKSON, 1993), a complexidade é influenciada pelo número de partes reconhecíveis, intensidade de interação e regras de organização entre as partes. Ruelle (1993) associa a complexidade de um sistema à força das interações presentes. Para Maximiano (1997), a complexidade em uma organização indica o número de situações e variáveis que gestores podem se deparar ao longo de seus processos. $\mathrm{O}$ autor caracteriza a complexidade em organizações por: (i) nenhum problema é totalmente linear; (ii) não existe efeito resultante de uma única causa nem uma causa que produza um único efeito; e (iii) quanto mais variáveis ativas, mais interdependências.

No pensamento complexo, é a não-linearidade e o papel criativo da desordem que explicam as trajetórias irreversíveis e as emergências, resultados imprevisíveis apresentados pelos sistemas adaptativos. Emergências são propriedades e capacidades ausentes nas partes, mas presentes quando estas se reorganizam, adaptando-se a um fator externo que desordena e modifica as relações vigentes no sistema. A desordem externa provoca uma nova e emergente ordem interna. Não se pode deduzir o comportamento emergente, nem pelo comportamento das partes nem pelo comportamento passado do sistema. O modo como as partes se reorganizam após eventos desordenadores é inesperado e imprevisível, resultando estados improváveis e rotas irreversíveis (HEYLIGHEN, 1988).

A complexidade de um objeto pode ser vista como um fenômeno quantitativo: é a quantidade de informação necessária para descrever as interações e interferências mútuas de interesse entre partes (BAR-YAM, 1997). A complexidade também reside na incerteza entre as interações e na quantidade de desordem e ordem que nelas convivem. Em estatística, chega-se à ordem das populações observando a desordem das amostras. Em complexidade, partes do objeto interagem e geram comportamentos sistêmicos contra-intuitivos, inexistentes nas partes isoladas, e que desaparecem na sua separação. Em síntese, não se entende a complexidade de um objeto por amostras ou pelo estudo de suas partes isoladas, mas do estudo de seu todo e das interações que surgem quando as partes são integradas (DEMO, 2002; MORIN e LE MOIGNE, 2000; RUELLE, 1993). Para Cardoso e Serralvo (2009), a abordagem da complewxidade propõe uma visão de totalidade da realidade de um objeto que procedimentos determinísticos não podem oferecer.

A compreensão de objetos complexos em diferentes áreas do pensamento tem encaminhado a formação de novas emergências transdisciplinares, valorizando principalmente interações e interferências das partes com o todo. Em organizações, estas interações e interferências se materializam sob a forma de metassistemas, ou sistemas de nível superior, que englobam subsistemas, de níveis inferiores, ao mesmo tempo em que os controlam, atribuem objetivos e dão sentido à hierarquia que se forma (CARDOSO e SERRALVO, 2009; GENELOT, 2002). 
Um dos objetivos atuais da ciência do gerenciamento é entender e explicar a realidade que surge quando empresas se organizam em sistemas de produção multiestágios, tais como ocorre em cadeias de suprimentos (SC) e em redes de cooperação (GENELOT, 2002). Entende-se que muito da estruturação, organização e elementos de gestão de cadeias de suprimentos e redes de empresas tenha sido adotado por estrategistas e organizadores de cadeias em função dos efeitos e do comportamento complexo observado nas principais variáveis. Dentre estas, pode-se apontar a produtividade, em unidades por período de tempo e principalmente o tempo de atravessamento de mercadorias e o inventário observado nas operações.

Um destes efeitos, observável em cadeias de suprimento, é o efeito-chicote. Este efeito se caracteriza por flutuações nas demandas internas (pedidos entre empresas da cadeia) muito maiores do que as variações nas demandas dos clientes finais. O comportamento complexo externo surge pela adoção de regras locais, tais como: a demanda do próximo período de uma parte é a previsão de demanda do período atual mais uma correção proporcional ao erro, usual em métodos de previsão de demanda consolidados nas empresas, tal como a média móvel exponencialmente ponderada.

Tal efeito se reflete em níveis médios e variabilidade de inventário muito mais elevados do que seria de esperar. Como consequiência das flutuações de inventário, também os tempos de atravessamento flutuam de modo oscilatório. Como efeito secundário, em função de eventuais faltas de matéria-prima causadas pela excessiva flutuação, a produtividade em peças entregues por período de tempo cai, observando-se resultado final menor do que o menor elo da cadeia. Este efeito surge, entre outras causas, pelo efeito sistêmico nas variáveis, originado de atrasos e realimentações internas. Diversas estruturas físicas e de gestão são observadas nas cadeias para lidar com este efeito, tal como redes de distribuição, compostas por armazéns e rotas de transporte mais articuladas e mais densas, uso de facilidades de concentração, tal como centros de distribuição e o uso de lotes menores, para antecipar o mínimo possível a demanda, pois esta poderá flutuar mais do que o necessário.

O efeito chicote é abundantemente tratado na literatura. Uma das obras que o trata é Simchi-Levi et al. (2003), incluindo análise matemática e uso de simulação. A obra cita outras referências.

O objetivo deste artigo foi apresentar uma técnica quantitativa para calcular a complexidade em arranjos organizacionais. O método de pesquisa foi o estudo de dois casos. Como são casos, as conclusões ainda não são generalizáveis. Foram calculadas a complexidade de uma cadeia de suprimentos e de uma rede de ateliers de costura da indústria calçadista, formada por pequenas e médias empresas. Uma análise comparativa de arranjos produtivos incluindo de cadeias de suprimentos e redes de pequenas e médias empresas é apresentada em Hansen e Oliveira (2009).

Ao fim, chegou-se a um indicador de complexidade para os arranjos, que poderá, eventualmente, ser usado como elo de realimentação e controle da complexidade do arranjo, segundo objetivos estratégicos de seus gestores. Uma aplicação anterior foi apresentada em Sellitto et al. (2010). Os autores usaram a técnica ora apresentada para calcular a complexidade de uma cadeia de suprimentos industriais da indústria metal-mecânica.

A gestão da complexidade é parte integrante da gestão da cadeia de suprimento e pré-requisito para a bemsucedida implementação do SCM no ambiente competitivo moderno. A gestão de complexidade não deve ser executada no nível da empresa, mas da cadeia, em cooperação integrada (KALUSA et al., 2006). Devido à ligação entre a complexidade e a eficiência da cadeia de suprimentos, a gestão da complexidade pode ser considerada como um desafio importante na gestão de hoje (BLECKERB e KERSTEN, 2006). Entende-se que, dentro da gestão da complexidade da cadeia de suprimentos, a existência de um modelo para a medição seja condição essencial para seu controle e o controle da complexidade é essencial para a sua gestão.

Este artigo dá continuidade à linha de pesquisa cuja revisão teórica completa foi apresentada em Sellitto et al. (2008b). Até então havia sido usado um método qualitativo, baseado em opiniões de gestores e estrategistas de arranjos de empresas (SELLITTO et al., 2007; 2008a). Agora, apresenta-se uma variante quantitativa do método, criando um modelo baseado em medições de campo das variáveis que podem apreender a complexidade da cadeia e do arranjo de empresas.

Por um lado, avaliações qualitativas apreendem bem impressões de especialistas sobre objetos complexos, mas apresentam subjetividade intrínseca no resultado. Por outro, medições baseadas em variáveis de campo apreendem apenas o valor da variável, mas são objetivas, não dando margem a interpretações. Entende-se que as variantes do método são complementares e desejáveis.

Os resultados de aplicações anteriores foram usados para comparação com os deste artigo.

O restante do artigo está organizado em: revisão menor sobre sistemas adaptativos complexos (a revisão completa foi publicada em Sellitto et al. (2008b)), fundamentos sobre medição de complexidade organizacional e relato da metodologia, resultados e discussão.

Parte da pesquisa foi financiada pelo CNPq. 


\section{Sistemas adaptativos complexos}

A ciência clássica tem tentado explicar o comportamento dos sistemas por aproximações lineares. $\mathrm{Na}$ relação linear, há um efeito para uma causa e uma causa para um efeito. O efeito combinado de duas causas é a soma dos efeitos separados de cada causa. A intensidade do efeito cresce segundo a intensidade da causa. Pode-se separar o sistema em partes, estudá-las isoladamente e recompô-las, sem perda de conteúdo (WIENER, 1970). $\mathrm{Na}$ relação não-linear, há incerteza e várias causas podem produzir o mesmo efeito: a mesma causa pode produzir mais de um efeito (PARKER e STACEY, 1995). Há sinergias e o resultado pode ser maior ou menor do que a soma das partes. Sistemas não-lineares são imprevisíveis, pois o resultado de uma ação não é garantido e uma aproximação reducionista só é válida se a sinergia for irrelevante.

Para Cardoso e Serralvo (2009), causalidades lineares e únicas do tipo tal causa produz tal efeito podem ser encontradas em áreas restritas das ciências físico-químicas. No entanto, em ciências sociais aplicadas, tais como a engenharia de produção, fenômenos multicausais podem produzir efeitos múltiplos. Pode ser, portanto, infrutífera a tentativa de se obterem causas únicas que expliquem efeitos únicos. Quando se tenta demonstrar que certos dados produziram certos resultados, geralmente não se demonstra que não existem outros dados capazes de produzirem o mesmo resultado. Em síntese, relações causais complexas não são descritas por fórmulas do tipo se p então $q$.

A teoria dos sistemas adaptativos complexos é herdeira da teoria de sistemas. Até quanto se sabe, a idéia de uma teoria geral de sistemas foi introduzida nos anos 1930 pelo biólogo austríaco Ludwig von Bertalanffy. A primeira apresentação oral do conceito foi feita por Bertalanffy em Chicago, em 1937, e as primeiras apresentações em artigos se deram entre 1945 e 1950 (Klir, 1969). Bertalanffy seguiu uma linha de pensamento trilhada por outros cientistas e filósofos que, desde Aristóteles, com a teleologia, e passando por Leibnitz e Kant, procuraram uma filosofia natural: um conjunto de explicações que descreva, com poucas adaptações, qualquer observação na natureza. As obras de Lotka, Whitehead e Cannon, nos anos 1920, entre outras, prenunciaram e subsidiaram tais idéias.

Bertalanffy $(1976$; 1977) elaborou o conceito de sistema ao perceber que idéias gerais referentes a todos organizados poderiam ser aplicadas a outros todos organizados. Para ele, um sistema é um complexo de elementos que operam relações ordenadas, não fortuitas. Elementos $p$ participam em relações $R$, de modo que o comportamento de $p_{1}$ em $R_{1}$ é diferente do comportamento de $p_{1}$ em $R_{2}$. Se os comportamentos de $p_{i}$ em $R_{i}$ não forem diferentes, não há interação mútua e então as relações $R_{i}$ independem dos elementos $p_{i}$. Hopeman (1977) classifica as relações em sistemas como: (i) de primeira ordem, se são vitais para o propósito do sistema, tal como o abastecimento de material em máquinas; (ii) de segunda ordem, se melhoram o desempenho de um elemento, tal como o treinamento do operador da máquina; e (iii) de terceira ordem, se aumentam a confiabilidade, tais como duas máquinas que podem cumprir a mesma tarefa. Para Checkland e Scholes (1999), um sistema é um todo complexo, cujas propriedades se referem ao todo e não às partes. Tais propriedades são: (i) emergentes, quando surgem do fato de diversas partes terem sido colocadas juntas no todo, tal como a capacidade de deslocamento que surge quando partes de uma bicicleta são agregadas; (ii) hierárquicas, se, a medida que se avança da base para o topo, varie seu alcance; e (iii) ligadas a sobrevivência, pois um todo só sobrevive em um meio variável se puder se auto-adaptar. Os autores propõem uma metáfora: um sistema é um conjunto adaptativo capaz de sobreviver em um ambiente mutável. Para Maciel (1974), um sistema é um todo organizado dinamicamente relacionado com o seu exterior, sujeito a mudanças permanentes e que apresenta a cada momento um modo diverso de ação ou comportamento. O autor destaca definições auxiliares: (i) transformada: é o novo estado de um sistema; (ii) transição: é a passagem de um estado a outro; (iii) transformação: é uma sequiência de transições; (iv) processo: é uma seqüência de transformações; (v) desenvolvimento: é uma seqüência de todos os processos; e (vi) evolução: é a sequiência de desenvolvimentos. Segundo Bauer (1999), um sistema tem um sentido ou propósito, é orgânico, mas tem partes interdependentes, pois reage a estímulos externos, mesmo se estes forem aplicados a apenas uma ou poucas partes.

O estudo de sistemas não-lineares ou complexos ou ainda adaptativos complexos tem sido aceito como disciplina unificada, resultante de esforços em campos tão díspares quanto as ciências naturais, a administração e a antropologia. Partindo da teoria geral de sistemas, a construção de uma teoria específica sobre sistemas adaptativos complexos tem como objetivo explicar a emergência da complexidade a partir de comportamento local simples e a emergência da simplicidade a partir de comportamento sistêmico complexo (BAR-YAM, 1997). Observam-se regularidades no comportamento não-linear ou complexo, independente da atomística do sistema: o princípio da auto-organização e a noção de emergência surgiram multidisciplinarmente em vários campos de pesquisa. Os proponentes da ciência da complexidade consideram que sistemas adaptativos complexos naturais ou artificiais, físicos, biológicos ou sócio-econômicos, podem ser caracterizados por um conjunto unificado de princípios que marcam uma mudança de paradigma no modo de explicar fenômenos e sistemas (BAR-YAM, 1997; MARKOSE, 2005; HEYLIGHEN, 1988). 
Para Agostinho (2003), a propriedade básica de sistemas adaptativos complexos é a capacidade de modificar comportamento a partir do que percebe quanto ao meio e seu próprio desempenho em atingir um propósito. Segundo a autora, padrões de comportamento observáveis não surgem de fora, mas da interação entre agentes autônomos internos. Gell-man (2000) fala em comportamento superficial complexo, originado de simplicidade interna profunda. Gleick (1990) aponta que o comportamento complexo pode surgir de regras locais simples. Bar-Yam (1997) fala em blocos básicos com os quais pode-se engendrar, a partir do simples, um sistema de comportamento complexo. Cadeias de suprimentos e redes de cooperação industriais apresentam tal comportamento, pois basta que cada membro, os blocos básicos, persiga objetivos requeridos por parceiros adjacentes para surgir um comportamento exterior difícil de decifrar a olho nu ou com os instrumentos usuais de controle gerencial. No sentido contrário, sistemas adaptativos de alta complexidade interna podem produzir alguns poucos padrões de comportamento externo, os atratores (LEWIN, 1994; GLEICK, 1990).

Agostinho (2003) sintetiza: sistemas adaptativos complexos são organizações de agentes autônomos e ativos, cuja ação é governada por regras de comportamento sujeitas a modificações e aprendizado por pressões do ambiente e informações de desempenho. O comportamento global emerge como efeito da combinação de interações não-lineares entre agentes ativos e componentes observáveis do sistema.

Sistemas adaptativos podem ser classificados segundo sua complexidade. Um continuum de complexidade contém, em uma extremidade, sistemas pouco adaptáveis, caracterizados por um pequeno número de elementos, com interações fracas, governados por leis claras e delimitadas, fechados ao ambiente e estáticos no tempo. Este tipo de sistema é pouco suscetível a influências e seus subsistemas não têm objetivos específicos. A outra extremidade do continuum contém sistemas com muitos elementos e fortes interações mútuas. Tais sistemas são probabilísticos, abertos e dependentes do ambiente, evoluem, diferenciam-se no tempo e seus subsistemas possuem objetivos próprios (JACKSON, 1993). Sistemas planetários estão na primeira extremidade, enquanto que sistemas humanos estariam na segunda. Sistemas de fabricação ou prestação de serviço estão em posições intermediárias, segundo seu mecanicismo ou organicismo.

Cadeias de suprimentos e redes de cooperação apresentam características de sistemas adaptativos complexos: empresas individuais produzem comportamento complexo quando, na busca por objetivos maiores compartilhados, perseguem objetivos locais intermediários, tais como melhorias em entregas, qualidade ou custos. Nestes arranjos, interações entre empresas formam subsistemas e hierarquias, como nos sistemas estudados em outras ciências (GENELOT, 2002). Duas principais fontes podem responder pela complexidade nestes arranjos: globalização de operações e heterogeneidade entre os membros. Entre outras causas, rotas de transporte mais longas, produtos mais sofisticados, lotes de produção menores, restrições ambientais suportam a primeira fonte. Entre outras causas, variedade e quantidade de membros, diferentes estratégias de marketing e de produção, diferentes sistemas de contabilidade e de medição de desempenho suportam a segunda fonte. A complexidade surge quando partes com tamanhas diferenças devem ser colocadas juntas para atingir objetivos comuns de negócios (SASSI, 2006). Em suma, complexidade surge devido às contínuas interações entre as partes causadas pela necessidade de trocas, de compartilhar recursos e de negociar objetivos comuns.

Cadeias de suprimentos e redes de cooperação podem ser consideradas sistemas de complexidade intermediária. Suas relações internas são impostas pela estratégia de gestão e são mais fortes e menos previsíveis do que em sistemas mecânicos, mas mais fracas e menos aleatórias do que em sistemas humanos organísticos (JACKSON, 1993).

\section{Informação e complexidade}

A palavra latina informare, da qual se originou a palavra informação, significava originalmente dar forma ou aparência a um objeto ou ainda apresentar, representar ou criar uma idéia ou noção sobre o objeto. Informação significa a colocação combinada de elementos ou partes segundo um esquema ou classificação. Sob esta forma geral, a informação pode ser entendida como uma forma de classificação de símbolos e das relações que surgem entre estes quando organizados segundo um objetivo. A informação é quantitativa, na medida em que se preocupa com a forma com que um conjunto de símbolos se organiza, independente da atomística específica do objeto de estudo. Também é qualitativa, na medida em que pode ser entendida como a capacidade de organizar um conjunto específico de símbolos, segundo uma inteligência processual ligada à qualidade do material a ser organizado (ZEMAN, 1970).

A noção de informação pode ser usada para responder o quanto de complexidade há em um sistema adaptativo complexo. Neste caso, é preciso saber quanta informação é exigida para descrevê-lo. Segundo Galbrait (1977, apud FLYNN e FLYNN, 1999), a complexidade de uma organização está diretamente relacionada à quantidade de processamento de informação requerido, resultante da incerteza nos ambientes externos e internos.

A teoria da informação quantifica a informação contida em uma mensagem e pode ser usada para quantificar 
a complexidade de um objeto organizacional. Se uma sequência de símbolos, ou mensagem, puder ser associada a um vetor de variáveis de estado representativo de um sistema complexo, então o conteúdo de informação deste vetor pode ser usado como medida da complexidade do sistema (BAR-YAM, 1997).

Seja uma mensagem $s=\left[s_{1}, s_{2}, \ldots, s_{n}\right]$. Se $s 1=0$ e o alfabeto é binário, $s 1 \neq 1$. Já se o alfabeto é hexadecimal, $\mathrm{s} 1 \neq[1,2, \ldots, \mathrm{F}]$, ou seja, há mais informação em um caractere que pode assumir dezesseis estados do que em um que pode assumir dois estados. Se a mensagem descreve um objeto, a quantidade de informação I(s) contida no objeto é dada pela Equação 1, na qual, $\Omega$ é o número de mensagens possíveis. Se há $k$ caracteres no alfabeto e as probabilidades forem uniformes, o conteúdo de informação é dado pela Equação 2 (BARYAM, 1997; BAUER, 1999). Outras distribuições são remetidas à continuidade.

$$
I(s)=\log _{2}(\Omega)
$$

$$
I(s)=n \cdot \log _{2}(k)
$$

A complexidade de um sistema é proporcional ao comprimento da mais concisa mensagem capaz de descrever suas regularidades, em uma dada escala e uma dada linguagem (GELL-MANN, 2000). Para chegar a esta mensagem, é necessário descrever o sistema do modo mais comprimido possível, separando as regularidades estruturais das aleatoriedades observadas no comportamento do sistema (RUELLE, 1993). GellMann (2000) e Heylighen (1988) sugerem grafos ou redes para tal. Anastasiadis et al. (2005) associam grafos a meias-matrizes, como foi feito neste artigo, para cálculo da complexidade em arranjos. A meia-matriz é essencialmente a tabulação de informações sobre associações ou correlações entre dois nós dentro do arranjo. Na literatura internacional, é usado o termo assortativity (ANASTASIADIS et al., 2005). Wilhelm e Hollunder (2007) classificam arranjos quanto à complexidade comparando a média das interações informacionais observadas em um dado arranjo com a que surge aleatoriamente em simulações. Os autores propuseram métodos baseados em informação para medição e caracterização da complexidade em arranjos. Kim e Wilhelm (2008) calcularam a complexidade de vários tipos de grafos por vários métodos. Bauer (1999) usa a noção de estrutura, decompondo o sistema em subsistemas, em estrutura multinivelar escalonada. Simon (1991) usa hierarquias do tipo caixas-dentro-de-caixas, modeláveis matematicamente por estruturas em forma de árvores, conectadas por interações. Por fim, Agostinho (2003) diz que sistemas mais complexos se originam da agregação de sistemas menos complexos, cujas fronteiras são demarcadas pela intensidade das interações, mais fracas fora do que dentro dos subsistemas.
Em síntese, são dois os elementos conceituais de interesse desta pesquisa, complexidade e informação. Observada a complexidade intrínseca de um sistema, esta pode ser mensurada pela informação necessária para descrever as interações que surgem no interior do sistema. Ou seja, a complexidade nasce pela quantidade e intensidade das interações internas e estas são apreendidas pelas informações trocadas. Esta estrutura analítica foi a base para a coleta e a análise dos dados nos casos estudados.

\section{A Pesquisa: método de trabalho e resultados}

Devido ao crescente uso de arranjos de produção multi-estágios, tais como cadeias de suprimentos e redes de colaboração, compostos por várias empresas que interagem entre si na busca de objetivos comuns, produzindo uma complexidade subjacente às operações, uma questão relevante passa a ser a noção quantitativa do quão complexo é o arranjo (BAUER, 1999). Entendese que a gestão do arranjo deva ser capaz de dizer: a complexidade é $n$ unidades, em um referencial de máxima complexidade igual a $m$ unidades. Também deve ser capaz de dizer: a complexidade desejada é $c$ unidades e, para obtê-la, que ações de controle devem ser tomadas. A presente pesquisa atende ao primeiro requisito.

Para Bar-Yam (1997), medir a complexidade de um sistema é um experimento específico, pois um método válido para um sistema pode não o ser para outro. Rocha Neto (2003) pondera que a medição da complexidade de um sistema deve considerar o número de partes e os seus graus de interação. Assumiu-se como um dos pressupostos de pesquisa que a complexidade é proporcional à quantidade de informação necessária para operar o arranjo. Mais interações requerem mais informações e acarretam mais complexidade no sistema. Outro pressuposto admitido é: a complexidade atual nasce do número de oportunidades de surgirem interações entre partes e do grau das interações que realmente surgem. Hopeman (1977) reconhece o pressuposto quando classificou as relações existentes em sistemas complexos, como já mencionado. Rocha Neto (2003) também reconhece o pressuposto, ao admitir que sistemas simples e complexos se diferenciam segundo os graus de interação que as partes apresentam. Outro pressuposto é: quanto mais intensa é uma interação, mais informações são necessárias para descrevê-la. Isto permite usar a teoria da informação, que mede a quantidade de informação em uma mensagem, para medir a complexidade que surge em uma interação, se esta for descrita como se uma mensagem fosse trocada entre as partes.

O objetivo de pesquisa é apresentar uma técnica quantitativa para avaliar a complexidade em arranjos 
organizacionais. Considerando as diversas perspectivas, já relatadas, mensurou-se a complexidade de dois sistemas adaptativos quantificando e somando o número de informações relevantes trocadas entre as partes para o gerenciamento da operação. Usaram-se grafos e meiasmatrizes, tal como mencionado na literatura. Chegou-se a um indicador de complexidade que pode ser usado como elo de realimentação para seu controle por ações gerenciais. Não é objetivo chegar a um indicador unificado para a complexidade de organizações produtivas, mas a um indicador complementar aos já citados na revisão, baseado nos pressupostos ora admitidos.

A questão de pesquisa foi: como medir a complexidade subjacente às operações de um arranjo interorganizacional, no qual diversas empresas trabalham juntas para a obtenção de um objetivo comum? Conforme Yin (2001), questões de pesquisa do tipo como podem ser respondidas por uma estratégia de pesquisa baseada em casos. O método de pesquisa escolhido foi, portanto, o estudo de caso. Optou-se pelo caso duplo: escolheramse dois sistemas complexos adaptativos, uma cadeia de suprimentos da indústria calçadista e uma rede de ateliers de facção da mesma indústria.

O estudo de caso descreve um fenômeno que, se repetido e observadas regularidades consistentes, pode gerar uma teoria fundamentada (EISENHARDT, 1989). A essência de um estudo de caso é a análise intensa de uma unidade de estudo, que pode ser um indivíduo ou um grupo de indivíduos, que não separa o fenômeno observado de seu ambiente (YIN, 2001). Segundo Eckstein (1975, apud ROESCH, 1999), há cinco modos com que um estudo de caso pode contribuir para uma teoria: (i) oferecer, para posteriores estudos, uma descrição profunda e específica de um objeto; (ii) interpretar eventuais regularidades como evidências de postulados teóricos ainda não enunciados; (iii) criar uma situação para testar uma ideia; (iv) sondar plausivelmente uma teoria proposta pelo terceiro modo; e (v) o caso crucial, que apóia ou refuta a teoria. Entende-se que a contribuição do presente estudo de caso é do terceiro tipo, uma situação heuristicamente construída para o teste de uma idéia.

O método de trabalho foi: (i) primeiro grupo focado com especialistas e pesquisadores que montaram uma estrutura de informações permanentes, não-aleatórias, que podem circular entre as empresas dos dois arranjos durante o gerenciamento; (ii) para cada arranjo, foi desenhado um grafo com as conexões regulares, não aleatórias; (iii) mais dois grupos focados com gestores dos arranjos que definiram os tipos de informação que são permanentes, não eventualmente, trocadas durante a operação; e (iv) foram calculadas as complexidades dos arranjos pela teoria da informação, usando a Equação 2 e $k=3$ (três possibilidades de resposta a cada tipo de informação: satisfaz, médio, insatisfaz). Outros alfa- betos são remetidos a continuidade. O uso de especialistas em grupos focados para modelagem e apoio à decisão é apresentado e explorado em Sellitto (2005).

\subsection{Resultados de pesquisa: estrutura para medição da complexidade}

O primeiro grupo de especialistas foi formado por dois gestores, um que atua na cadeia de suprimentos, outro na rede, com passagem por empresas da indústria calçadista; um profissional autônomo consultor de logística empresarial que atua na mesma indústria; e dois pesquisadores com experiência empresarial e atividade acadêmica também na indústria calçadista. Os participantes não-acadêmicos têm expressiva experiência empresarial e formação superior compatível (engenharia e administração, todos com mestrado). Sob a mediação dos pesquisadores, o grupo listou assuntos que podem ser considerados como comunicações permanentes necessárias de ser mantidas em arranjos como os estudados. O fio condutor da discussão foram perguntas em complexidade crescente, conforme sugerido em Ribeiro e Newmann (2003). Por análise de discurso dos participantes, os pesquisadores compuseram a estrutura de informações mínimas necessárias para gerenciamento na indústria calçadista. Ao fim, os praticantes criticaram e refinaram a estrutura. Devido à fenomenologia presente na pesquisa, a estrutura de informações obtida é válida só na indústria calçadista, no momento da pesquisa e na perspectiva dos participantes. Outra indústria, outro momento ou outros participantes possivelmente produziriam outra estrutura.

A manufatura de calçados é um processo intensivo em mão-de-obra: corte, costura, pré-fabricação, montagem e acabamento. $\mathrm{O}$ corte recebe matérias-primas, moldes e navalhas de fornecedores. Na costura, as peças são unidas por colagem ou costura. Na pré-fabricação, ocorre a fabricação e acabamentos em solas, saltos e palmilhas. Na montagem, o cabedal é montado sobre a fôrma e a sola é colada. No acabamento ocorrem operações como a escovação, limpeza, colocação de produtos auxiliares para embalagem, inspeção final e embalagem em caixa individual e coletiva. Corte, costura e pré-fabricação ocorrem interna e externamente, em ateliers, e são abastecidos por fornecedores de materiais e ferramentas, formando uma rede de operações, sincronizadas pela montagem.

A estrutura a que os participantes chegaram é mostrada no Quadro 1.

A estrutura é composta por seis grandes temas, que foram desdobrados em vinte tipos de informação que podem ser trocadas permanentemente, não aleatoriamente: previsão e programação de demanda; operação logística; pessoal; processos de gestão; finanças; e tecnologia e inovação. 
Para previsão de demanda dos diversos membros do arranjo, usualmente a gestão usa algoritmos ou heurísticas que se valem de informações de desempenho passado de vendas e da capacidade instalada nos diversos membros. Para a operação logística, usualmente a gestão precisa conhecer a programação das diversas entregas de produto acabado que o arranjo deve fazer, os diversos recebimentos de matéria-prima externa, a situação dos estoques em mãos, a situação dos materiais que podem ser compartilhados entre membros do arranjo e o compartilhamento de cargas e transportes, principalmente segundo a lógica milk-run, usada no interior dos arranjos. Quanto a pessoal, o arranjo troca informações sobre a disponibilidade e a habilitação de operadores, as necessidades e possibilidades de treinamento interno e eventual reaproveitamento entre empresas e treinamento e educação externas. Pelo porte que a operação conjunta adquire, o arranjo pode influenciar escolas e entidades

\begin{tabular}{cc}
\hline Temas & Tipos de informação trocadas \\
\hline Previsão e programação de demanda & Informações sobre desempenho de venda de produtos \\
Situação de capacidade instalada & Programação de entregas \\
& Programação de recebimentos \\
Pessoal & Situações de estoques e materiais compartilhados \\
& Compartilhamento de cargas e de transportes \\
& Disponibilidade e habilitações de operadores \\
Processos de gestão & Treinamentos e educação externos compartilhados \\
& Treinamentos internos compartilhados \\
& Situação de indicadores e de objetivos estratégicos \\
& Compartilhamento de processos de compra \\
Finanças & Compartilhamento de processos de venda \\
& Visita conjunta a feiras \\
& Situação de fluxos de caixa \\
Tecnologia e inovação & Adiantamento de pagamentos e empréstimos \\
& Financiamentos compartilhados \\
& Investimentos compartilhados em tecnologia \\
& Compartilhamento de máquinas e equipamentos \\
& Transferência de tecnologia \\
\hline
\end{tabular}

Quadro 1 - Informações que podem ser trocadas entre empresas em arranjos na indústria calçadista

educacionais da região a formatar atividades educacionais de interesse. Quanto aos processos de gestão, os membros podem trocar informações sobre a situação de indicadores, principalmente aqueles ligados aos objetivos estratégicos, tais como percentual de refugos, custo de componentes etc. Processos de compra e venda e visitas a feiras também são compartilhados. Em finanças, interessa conhecer o fluxo de caixa das empresas, para prevenir falta de caixa, as necessidades e disponibilidades de empréstimos ou adiantamentos entre empresas e eventuais possibilidades e disponibilidades de financiamentos compartilhados. Por fim, quanto a tecnologia e inovação, é de interesse comunicar os novos produtos e as modificações que ocorrem nos produtos existente e explorar possibilidades de investimentos conjuntos em tecnologia e equipamento e eventuais trocas de tecnologias entre as empresas.

\subsection{Resultados: o primeiro caso}

O primeiro caso foi estudado em uma empresa focal que monta calçados infantis e produz acessórios para varejistas multimarcas e lojistas franqueados. A empresa opera em três sedes, distantes mais de cem quilômetros entre si e dependente de sistema de informação para a gestão do arranjo. A capacidade total do arranjo produtivo pode chegar a 16.000 pares diários e depende de fornecedores de materiais e serviços. $\mathrm{O}$ sistema complexo adaptativo estudado foi a cadeia de suprimentos (SC - supply chain) da empresa focal. As informações sobre a estrutura da cadeia foram obtidas pelos pesquisadores em reunião com o membro do grupo focado anterior que atua como executivo da empresa e com o consultor de logística.

A SC conta com operações de fornecimento, transporte e armazenagem. A empresa focal é a manufatura $\mathrm{M}$, que recebe componentes e atende revendedores. Os principais membros da SC são: (i) couro (C); (ii) material sintético e têxtil para cabedal (S1 e S2); (iii) materiais de reforço para cabedal (R1 e R2); (iv) materiais metálicos de enfeite (E); (v) Adesivos (A); (vi) varejistas multimarcas (V); e (vii) varejistas franqueados (F). A também fornece adesivos para R1, R2, S1, S2 e P. A 
relação com os varejistas multimarcas V são simples transações comerciais. Com os varejistas franqueados F há mais troca de informações mercadológicas e gerenciais que configuram parceria mais intensa. As empresas S1 e S2 e R1 e R2 são concorrentes entre si. A Figura 2 mostra um grafo representativo da parte da SC considerada no estudo.

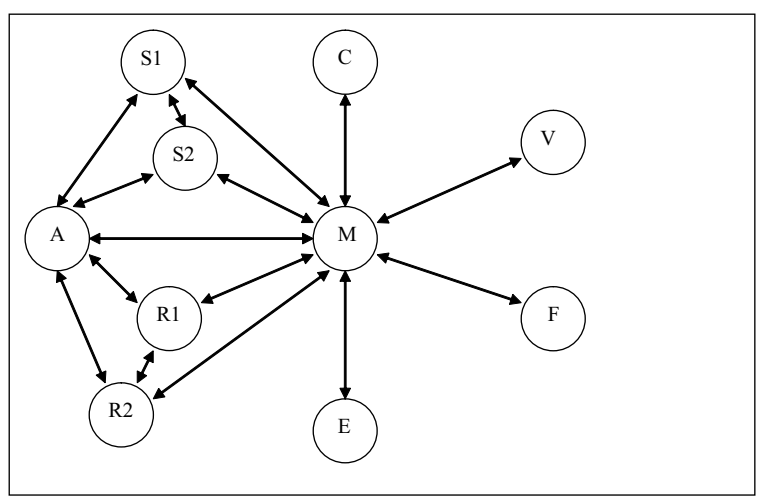

Figura 1 - Grafo representativo da parte da SC considerada

De posse do Quadro 1 e da Figura 1, os pesquisadores formaram novo grupo focado, com a presença de trê gestores da empresa, responsáveis respectivamente por compras, fabricação e logística de entrega. Todos possuem experiência na indústria e na empresa e formação superior compatível (engenharia e administração). Os pesquisadores estimularam o grupo a identificar, dentre as possibilidades do Quadro 1, quais os tipos de informação que precisam ser permanentemente trocadas entre os membros constantes do grafo da Figura 1. Para exemplificar, entre a empresa focal $\mathrm{M}$ e o fornecedor de materiais de enfeite $\mathrm{E}$, dos vinte tipos de informações possíveis, são trocadas permanentemente seis: (i) informação sobre desempenho de vendas de produtos; (ii) programação de entregas; (iii) situações de estoques e materiais compartilhados; (iv) treinamentos e educação externos compartilhados; (v) novos produtos e modificações em produtos existentes; e (vi) compartilhamento de máquinas e equipamentos. Eventualmente, outros tipos de informação podem ser trocados, mas de modo aleatório, não permanente, não sendo considerados para a medição de complexidade objetivada. O processo foi repetido para as demais empresas do arranjo.

Os resultados estão na Tabela 1.

Tabela 1 - Medição da complexidade na operação da SC

\begin{tabular}{|c|c|c|c|c|c|c|c|c|c|c|c|}
\hline & \multicolumn{10}{|c|}{ membros da cadeia } & \multirow[b]{2}{*}{ Soma } \\
\hline & $\mathrm{M}$ & $\mathrm{C}$ & $\mathrm{S} 1$ & $\mathrm{~S} 2$ & $\mathrm{~A}$ & $\mathrm{R} 1$ & $\mathrm{R} 2$ & $\mathrm{E}$ & V & $\mathrm{F}$ & \\
\hline Empresa focal M & & 9 & 8 & 8 & 9 & 5 & 5 & 6 & 6 & 15 & 71 \\
\hline Fornecedor C & & & 2 & 2 & 3 & 0 & 0 & 0 & 0 & 0 & 16 \\
\hline Fornecedor S1 & & & & 7 & 6 & 0 & 0 & 0 & 0 & 0 & 23 \\
\hline Fornecedor S2 & & & & & 6 & 0 & 0 & 0 & 0 & 0 & 23 \\
\hline Fornecedor A & & & & & & 3 & 3 & 3 & 0 & 0 & 33 \\
\hline Fornecedor R1 & & & & & & & 7 & 0 & 0 & 0 & 15 \\
\hline Fornecedor R2 & & & & & & & & 0 & 0 & 0 & 15 \\
\hline Fornecedor E & & & & & & & & & 0 & 0 & 9 \\
\hline Varejo Multimarca V & & & & & & & & & & 1 & 7 \\
\hline Varejo Franquia F & & & & & & & & & & & 16 \\
\hline & & & & & & Total d & inforn & ões & npart & das $=$ & 228 \\
\hline & & & & & & & & & & dia $=$ & 22,8 \\
\hline & & & & & & & esvio $\mathrm{p}$ & lrão & distri & ção $=$ & 18,50 \\
\hline & & & & & & & & ficier & de va & ção = & 0,81 \\
\hline & & & & & & Máx & no de & form & es pc & veis $=$ & 1.800 \\
\hline & & & & ntids & de il & formaç & atual & com & xidac & tual $=$ & 108,8 \\
\hline & & & Quantid & e de & orma & çao má & $\mathrm{ma}=\mathrm{c}$ & nples & ade $n$ & $\operatorname{ma}=$ & 858,8 \\
\hline & & & & & & & & mple & lade & $\operatorname{tiva}=$ & $12,67 \%$ \\
\hline
\end{tabular}


As células contêm quantos tipos de informação são trocadas entre o membro na linha e o membro na coluna. A última coluna apresenta o total de troca de informações em que um membro toma parte. Para esta soma, devese considerar a participação do membro como coluna e como linha, pois as informações são bilaterais. Por exemplo, o varejo multimarca $\mathrm{V}$ participa em sete trocas, seis com a empresa focal e uma com o varejo franquia. A meia-matriz resultante é consistente com representações de arranjos considerados complexos, como apresentado em Claussen (2007) e com as considerações apresentadas em Anastasiadis et al. (2005) sobre associações ou correlações (assortativity) entre dois nós dentro do arranjo.

O total de tipos de informação trocadas no arranjo é de 228 e o máximo possível é 1.800 (dez empresas podem trocar até vinte tipos de informação com outras nove). A complexidade atual é de 108,8 (Equação $2 \operatorname{com} n=$ 228 e $k=3$ ), a complexidade máxima é de 858,8 ( $n=$ $1.800)$ e a complexidade relativa é de $12,67 \%$. A distri- buição das trocas de informação pode informar sobre a governança. M troca 65 tipos de informações, enquanto a média é de 22,8, apontando a condição de elemento influente. $\mathrm{O}$ coeficiente de variação $c v=\sigma / \mu$ é igual a 0,81 .

\subsection{Resultados: o segundo caso}

O segundo sistema complexo estudado é uma rede de ateliers que prestam serviços de costura para uma empresa de componentes de calçados. São empresas de micro ou pequeno porte, geralmente familiares e de gestão menos profissionalizada. Os proprietários, muitas vezes, são ex-funcionários das grandes fábricas de calçados que adotaram a estratégia de terceirização para redução de custos fixos. A costura é sazonal, o que faz com que muitos contratos entre empresas e ateliers sejam de curto prazo.

A Figura 2 traz o grafo que ilustra o sistema e suas relações.

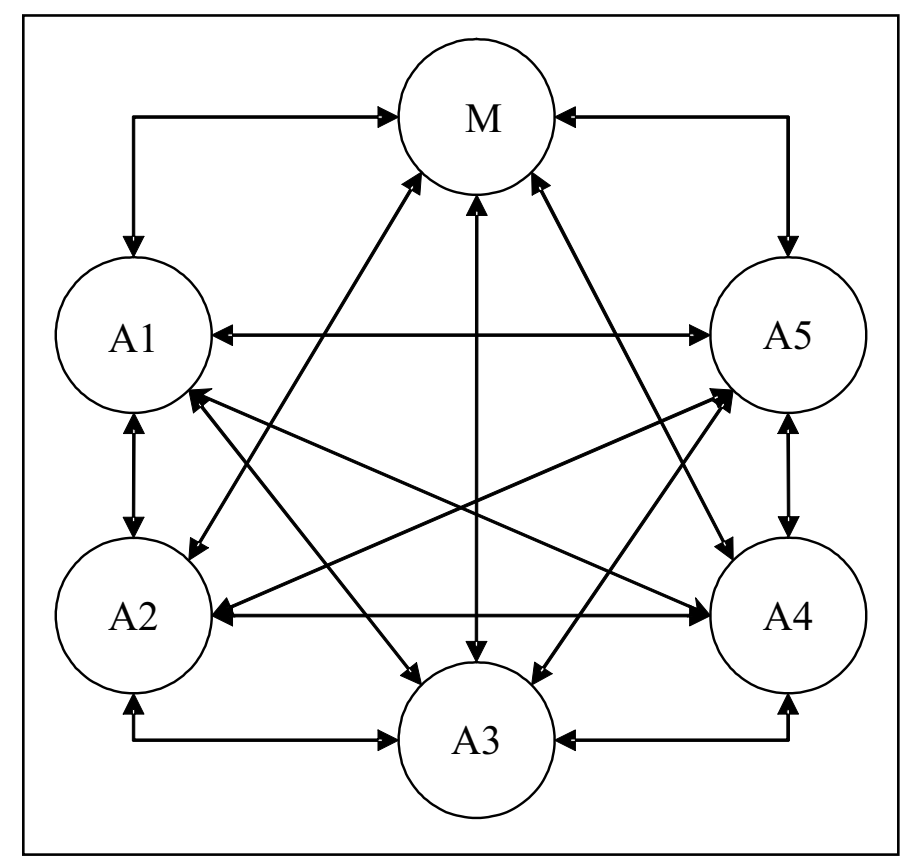

Figura 2 - Grafo da rede de ateliers

O sistema é composto por cinco ateliers A que cooperam e competem pelo mesmo tipo de serviço para a empresa focal M. A empresa focal não garante serviço contínuo para todos ao mesmo tempo, portanto qualidade, pontualidade e preço são fatores de decisão na rede. É necessária cooperação, pois dificilmente um atelier pode assumir sozinho a totalidade do serviço demandado. É necessário que vários ateliers trabalhem em multi-estágios produtivos, um fornecendo material para o outro, sem passar pela empresa focal. Existe plena autonomia entre os ateliers para que conversem e formulem estratégias de atendimento a $\mathrm{M}$, acarretando comunicação e troca de informações entre as partes.
Este sistema de fornecimento foi criado por M com o objetivo de terceirizar a produção de uma atividade que era caracterizada pela excessiva variação de demanda. Muitas vezes a empresa focal intervém no sistema, dando aporte de gestão e até mesmo financeiro para os ateliers, afim de estabilizá-lo.

O processo de análise das informações trocadas entre os indivíduos do sistema seguiu metodologia idêntica ao caso 1 . O grupo focado foi composto por um gestor da empresa focal, pelo mesmo consultor de logística do caso 1 e um gestor de atelier. Os resultados encontrados são apresentados na Tabela 2. 
Tabela 2 - Medição da complexidade na operação da rede

\begin{tabular}{|c|c|c|c|c|c|c|c|}
\hline & \multicolumn{6}{|c|}{ membros da rede } & \multirow[b]{2}{*}{ Soma } \\
\hline & $\mathrm{M}$ & A1 & A2 & A3 & A4 & A5 & \\
\hline Empresa focal M & & 9 & 8 & 8 & 9 & 5 & 39 \\
\hline Atelier A1 & & & 2 & 2 & 3 & 0 & 16 \\
\hline Atelier A2 & & & & 7 & 6 & 0 & 23 \\
\hline Atelier A3 & & & & & 6 & 0 & 23 \\
\hline Atelier A4 & & & & & & 3 & 27 \\
\hline \multirow[t]{9}{*}{ Atelier A5 } & & & & & & & 8 \\
\hline & \multicolumn{6}{|c|}{ Total de informações compartilhadas $=$} & 136 \\
\hline & \multicolumn{6}{|c|}{ Média $=$} & 22,7 \\
\hline & \multicolumn{6}{|c|}{ Desvio padrão da distribuição $=$} & 10,4 \\
\hline & \multicolumn{6}{|c|}{ Coeficiente de variação $=$} & 0,46 \\
\hline & \multicolumn{6}{|c|}{ Máximo de informações possíveis = } & 600 \\
\hline & \multicolumn{6}{|c|}{ Complexidade atual $=$} & 64,9 \\
\hline & \multicolumn{6}{|c|}{ Complexidade máxima $=$} & 286,3 \\
\hline & \multicolumn{6}{|c|}{ Complexidade relativa $=$} & $22,67 \%$ \\
\hline
\end{tabular}

O total de tipos de informação trocadas no arranjo é de 136 e a máxima quantidade de informação possível é 600 (cada uma das cinco empresas pode trocar até vinte tipos de informação com outras cinco). A complexidade atual é de 64,9 (Equação 2 com $\mathrm{n}=136$ e $\mathrm{k}=$ 3), a complexidade máxima é de $286,3(\mathrm{n}=600)$ e a complexidade relativa é de $22,67 \%$. A empresa focal troca 39 tipos de informações, enquanto a média é de 22,7 . O coeficiente de variação $c v=\sigma / \mu$ é igual a 0,46 .

\section{Discussão}

Inicialmente, foram discutidos aspectos ligados ao método e a conceitos da teoria da complexidade. Após, aspectos numéricos dos casos, inclusive comparando com casos anteriores. Por fim, foram feitos comentários sobre estratégia observada nos arranjos. Ressalte-se que, como são casos, as conclusões não devem ser generalizadas, apenas usadas como hipóteses para mais estudos, até que se chegue a dados suficientes para enunciados teóricos.

Observaram-se os três princípios da complexidade (MORIN e LE MOIGNE, 2000). A dialógica surge na aleatoriedade com que demandas chegam aos arranjos, mas ao chegarem, há determinismo no atendimento (ordem e desordem). A recursão está na essência dos arranjos. Grupos sociais demandam produtos industriais e serviços especializados, o que produziu a SC e a rede de ateliers. Estas atraem trabalhadores que renovam e acrescem os grupos sociais que as produziram. O princípio hologramático também é observado, já que operações presentes no arranjo também estão presentes nas partes. Em cada parte da SC há todos os princípios da mesma: atender a um cliente interno por meio de recebimento de materiais, energia e informação, operações de recebimento, manufatura, distribuição e manutenção de máquinas, assim como a SC como um todo atende a clientes externos.

Os dois casos apresentam características de sistemas adaptativos complexos, pois são formados por partes individualizáveis que interagem, e que quando submetidas a interferências externas, podem surgir emergências que alteram a configuração original para novamente atingir o equilíbrio. A complexidade varia em função da quantidade de interações regulares presentes. São sistemas abertos que retiram do meio os recursos de produção e retornam produtos acabados e resíduos. São realimentados por informações e têm a capacidade de se auto-reorganizarem, em resposta a forças externas ao sistema, como falta de alguma matéria-prima, variações nas demandas de clientes ou chegada de entrantes. Os grafos usados nos casos são similares ao método indicado por Gell-Mann (2000) para ambientes intermediários entre ordem e desordem. Tais ambientes exibem regularidade suficiente para a auto-organização, mas não a ponto que não haja mais incertezas, que darão ao sistema as chaves para melhorar sua organização.

A SC apresenta aspectos de estrutura dissipativa, pois, se surge um desequilíbrio sensível em variáveis de estado, tais como níveis de estoque, produtividade ou prazos de entrega, a SC se transforma e se adapta à situação. Há dissipação de energia na forma de esforço de engenharia e de gestão, até que emergir a nova forma, muitas vezes segundo trajetória de difícil reversão. Como exemplo 
desta reorganização, pode ser citada uma ação conjunta conduzida pela empresa de adesivo A e a de enfeites E. Para resolver um problema no processo produtivo de $\mathrm{M}$, ambas conduziram um projeto de pesquisa em conjunto. Houve crise no abastecimento de um item fabricado por S1, que recorreu a S2. Houve falta de enfeites e M mudou o projeto e E o processo, encontrando um novo ponto de equilíbrio. Uma significativa alteração deu-se quando entrou no mercado local uma grande rede de lojas de departamento que só trabalha com artigos de cadeia concorrente. Houve sensível redução no volume de vários itens. Algumas empresas fornecedoras tiveram que encontrar outros clientes para reencontrarem seu equilíbrio. Fatores relativos ao ambiente, como a moda, podem determinar a capacidade produtiva do fornecedor de couro $\mathrm{C}$, pois a utilização desta matéria-prima varia de estação para estação, conforme as tendências.

No segundo caso, uma parte dos cinco ateliers colabora para fornecer a composição desejada de produto pela empresa focal. Se um dos ateliers não tem capacidade para fornecer sua parte, os outros assumem parcelas de responsabilidade, sendo admitido até a entrada temporária de um atelier de fora do arranjo. Existe dissipação sensível de energia no processo, sob a forma de esforço gerencial e logístico. A noção de autopoiese está presente na rede, pois a grande maioria dos ateliers é comandada por ex-funcionários e emprega mão de obra treinada e originada na empresa focal. Ou seja, há autoprodução de recursos orgânicos.

Passa-se aos resultados numéricos.

O número médio de interações por parte está associado à autonomia presente no arranjo e a variabilidade na distribuição ao formato auto-organizante adotado pela gestão do arranjo. Quando a autonomia cresce, diminui o número médio de interações. No extremo, quando a necessidade de interação é nula, a parte, além de autopoiética, pois produz a si mesmo, é totalmente autônoma para cumprir sua missão, pois não depende de outras partes. Quando a importância de uma parte sobre as outras cresce, cresce a variabilidade da distribuição, pois esta empresa forma uma assimetria nas comunicações, concentrando desproporcionalmente os canais de decisão (SELLITTO et al., 2008b).

A técnica auto-organizante concentrou mais poder na $\mathrm{SC}$ do que na rede e remete ao conceito de indivíduo influente (GELL-MANN, 2000). Este, se existir, dirige a evolução do sistema e o comportamento das partes, formulando estratégias e impondo restrições, como parece se dar mais na $\mathrm{SC}$ do que na rede, pois a manufatura $\mathrm{N}$ da SC exerce uma influência assimétrica em relação às outras partes, podendo criar restrições e formular a estratégia, mais do que $\mathrm{M}$ da rede, pois esta depende mais da disponibilidade e da capacidade dos membros da rede para formular a estratégia de produção.

$\mathrm{Na} \mathrm{SC}$ e na rede, o número médio de interações por parte é próximo (22,7 e 22,8), o que pode indicar autonomias equilibradas. Já o coeficiente de variação difere: 0,81 na SC, 0,46 na rede, o que indica distribuição de interações mais uniforme na rede e mais concentrada na $\mathrm{SC}$ e por conseqüência mais governança concentrada em poucas partes na $\mathrm{SC}$ do que na rede. $\mathrm{Na} \mathrm{SC}$, mais de $45 \%$ das informações do sistema passam por $\mathrm{M}$ e A, que são os maiores responsáveis por ampliar ou reduzir a complexidade. Por exemplo, a empresa de adesivo A participa do processo da empresa focal e tem acesso a informações de outros fornecedores e de clientes. Um exemplo desta governança é em relação ao contato das empresas de varejo $\mathrm{F} \mathrm{e} \mathrm{V} \mathrm{com} \mathrm{as} \mathrm{empresas} \mathrm{fornecedoras.}$ A empresa $\mathrm{M}$ concentra as informações de ambas as partes e tem o poder de decidir se os coloca em contato direto ou não. A elevada variabilidade significa que a troca de informações entre os outros indivíduos do sistema é baixa. No caso da rede, embora haja alguma assimetria em favor da empresa focal, não há tamanha concentração de governança, pois muitas informações são compartilhadas pelos ateliers, principalmente em relação às atividades de produção.

O número de interações potenciais na rede é menor do que na SC (600 x 1.800), mas este número é afetado pelo tamanho do arranjo ( 5 x 10 partes). A complexidade relativa não depende do tamanho do arranjo e é menor na SC do que na rede $(12,67 \%$ x 22,67\%). Estes resultados decorrem das definições estratégicas tomadas no projeto de gestão dos arranjos.

Os achados da presente pesquisa são complementares a achados anteriores e, em conjunto, permitem discussão que ultrapassa o atual escopo e assim deve ser considerado para o entendimento da discussão que segue.

Para análise estratégica, foram usados achados de casos anteriores dentro da mesma linha de pesquisa (SELLITTO et al., 2007, 2008a, 2010). Três parâmetros, o número médio de interações, o coeficiente de variação da distribuição e a complexidade relativa, foram considerados na comparação. Outros parâmetros são afetados pelo tamanho dos arranjos e não foram usados.

Os valores são sintetizados na Tabela 3. Os parâmetros das SC's calçadista e metal-mecânica 2 e rede de ateliers foram calculados pelo método quantitativo ora apresentado. Os demais, por método qualitativo: a avaliação do grau de interação entre as partes foi por meio de julgamento categórico de gestores e consultores especialistas. 
Sellitto, M. A.; Guimarães, M. G.; - Cálculo da Complexidade Organizacional em Dois Arranjos..

Tabela 3 - Comparação entre parâmetros de complexidade de arranjos

\begin{tabular}{ccccc}
\hline & $\begin{array}{c}\text { Método de } \\
\text { cálculo }\end{array}$ & $\begin{array}{c}\text { Média de } \\
\text { interações }\end{array}$ & $\begin{array}{c}\text { Coeficiente de } \\
\text { variação }\end{array}$ & $\begin{array}{c}\text { Complexidade } \\
\text { relativa }\end{array}$ \\
\hline SC calçadista & quantitativo & 22,8 & 0,81 & $12,67 \%$ \\
SC metal-mecânica 1 & qualitativo & 12,51 & 1,1 & $11,25 \%$ \\
SC metal-mecânica 2 & quantitativo & 21,1 & 0,83 & $13,20 \%$ \\
Rede ateliers & quantitativo & 22,7 & 0,46 & $22,67 \%$ \\
Rede de saúde & qualitativo & 9,33 & 0,32 & $20,75 \%$ \\
\hline
\end{tabular}

Algumas regularidades podem ser observadas. As SC's têm coeficiente de variação maior e complexidade relativa menor do que as redes. As médias de interações que foram calculadas por método quantitativo foram maiores e não apresentaram, ao menos até quanto se examinou, um padrão útil na análise. Wilhelm e Hollunder (2007) falam em arranjos mais democráticos, quando a importância das partes, associada a distribuição de interações, é maior, e arranjos ditatoriais, nos quais há mais concentração de interações em menos partes. Observando esta distinção, Sellitto et al. (2007) propuseram duas classes de estratégia para a análise de arranjos com base na complexidade relativa observada e no coeficiente de variação: responsividade e foco. No Quadro 2, foram sintetizadas as características observadas pelos autores nos arranjos estudados.

Quadro 2 - Relação entre atributos e estratégias presentes nos arranjos

\begin{tabular}{ccc}
\hline & \multicolumn{2}{c}{ estratégia } \\
\cline { 2 - 3 } atributo & responsividade & foco \\
\hline complexidade & reduz & aumenta \\
variedade entre as partes & reduz & aumenta \\
variedade dentro & aumenta & reduz \\
das partes & aumenta & reduz \\
custo de operação & reduz & aumenta \\
custo de transferências & & \\
e coordenação &
\end{tabular}

A estratégia de responsividade foi observada nas duas redes, associada a complexidade mais alta. Seu principal objetivo foi garantir agilidade na operação, de modo que o menor número possível de demandas, mesmo as imprevistas, fique sem resposta, e no menor tempo possível. Para tanto, admite redundâncias entre as partes, pois a mesma tarefa pode ser feita por mais de um indivíduo. Com isto, aumenta a variedade dentro das partes e diminui entre as partes, pois cada uma delas possui várias características diferentes e repetidas. A conseqüência é o aumento da confiabilidade da rede, ou seja, a probabilidade de que uma tarefa, mesmo inesperada, será atendida pela rede no tempo demandado. Estas escolhas aumentaram a complexidade da rede e o custo de operação, pois pode haver ociosidade: nem sempre haverá tarefas para todas as especialidades existentes em todas as partes. $\mathrm{O}$ custo das transferências e da coordenação cai, pois cada parte pode fazer maior percentual da atividade total.

A estratégia de foco foi observada nas SC's, associada a complexidade mais baixa. Seu principal objetivo foi garantir o menor custo possível de operação. O objetivo estratégico de redução de custo é alcançado pela focalização de atividades, concentração de especialidades e aumento no tamanho de lotes de produção, aumentando a especialização das partes e o enfoque. Cada parte faz uma e apenas uma atividade, com ocupação máxima e por consequiência ganho de escala e custo mínimo. Com isto, reduz a variedade dentro das partes e aumenta entre as partes, pois há poucas características diferentes e repetidas. A estratégia reduz a probabilidade de que uma ordem inesperada encontre um recurso disponível para atendimento imediato, mas aumenta a probabilidade de que seja atendida dentro de um prazo planejado e a mais baixo custo, pois concentra atividades e reduz ociosidades. Estas escolhas aumentaram a dificuldade de coordenação e concentraram a governança, aumentando o custo gerencial mas reduzindo o custo operacional. Por consequiência da concentração, reduziu a complexidade. No balanço, pode-se esperar que cadeias de suprimentos possam praticar preços mais baixos do que outros arranjos produtivos, o que é coerente com a noção de que custos em cadeias aumentam de modo não-linear com a complexidade (KALUSA et al., 2006).

\section{Considerações finais}

O objetivo deste artigo foi apresentar uma técnica quantitativa para cálculo da complexidade presente na gestão de arranjos organizacionais. Para tal, a pesquisa enumerou as interações presentes em dois arranjos de uma mesma indústria e usou a teoria da informação para calcular a complexidade. Aplicações anteriores constaram de avaliação qualitativa para mensurar a complexidade em uma cadeia de suprimentos industriais da indústria metal-mecânica e uma rede de postos de saúde de uma capital brasileira. Esta aplicação mensurou a complexidade de arranjos por método quantitativo. 
Em arranjos organizacionais, a complexidade surge da diversidade de especialidades, conhecimentos e talentos, o que produz redundâncias e variedades e permite a adaptação a realidades emergentes. Quanto mais variedade, mais complexidade. A estratégia de um arranjo organizacional pode explorar a diversidade na cognição, aprendizado, formulação e execução de estratégias. Ao contrário de um programa, que segue uma marcha fixa, uma estratégia lida com situações imprevistas e elementos que se modificam segundo informações emergentes e deve ser tratada por elementos que agreguem variedade ao arranjo.

Como conclusão da pesquisa, chegou-se ao seguinte resultado: a complexidade medida na cadeia de suprimentos foi de $12,67 \%$, enquanto que a da rede de empresas foi de $22,67 \%$. O resultado atual confirma resultados anteriores em que cadeias de suprimentos industriais mostraram valor de complexidade menor do que uma rede de empresas prestadora de serviços.

Acredita-se que o índice de complexidade a que se chegou possa ser útil para gestores de arranjos, pois pode ser aumentado ou reduzido por ações de alavancagem, segundo objetivos estratégicos. Para aumentar ou diminuir a complexidade, deve-se observar que partes do índice contribuem menos ou mais para o resultado final e tratálas. No dizer de Agostinho (2003, p. 17), "Decerto faltam bons modelos para entendermos o comportamento das organizações como sistemas complexos adaptativos". Quanto a SC's, Perona e Miragliota (2004) argúem que falta na literatura um modelo para o gerenciamento da complexidade nestes arranjos. Os autores apontam que a pesquisa tem enfocado parcialmente o problema, limitando-se a considerar poucas variáveis, tais como o número de produtos ou de fornecedores presentes no arranjo, para avaliações e gerenciamento da complexidade.

Talvez o modelo de análise ora proposto, se mais robustecido e refinado por mais aplicações, possa ser útil na descrição de organizações como sistemas adaptativos complexos e com isto colocar à disposição dos estudiosos de organizações o referencial teórico da teoria da complexidade.

Uma sugestão de continuidade é usar alfabetos mais numerosos e outras distribuições de probabilidade para suas incidências. Também sugere-se considerar interações superiores à segunda ordem e incertezas no grau da interação, incerteza nas intensidades das interações e o uso de simulação computacional para verificar a fronteira do caos em redes de cooperação e cadeias de suprimentos, o que demanda mais referencial teórico. Caso seja possível descrever variáveis de estado do arranjo como função das conexões, variando-as, é possível verificar como se comportariam no limite do caos. Por fim, novas pesquisas devem incluir outros tipos de arranjos interorganizacionais, tais como redes de inovação e distritos industriais.

\section{Referências}

AGOSTINHO, M. Complexidade e organizações. S. Paulo: Atlas, 2003.

ANASTASIADIS, A.; COSTA, L.; GONZALES, C.; HONEY, C.; SZELIGA, M.; TERHESIU, D. Measures of Structural Complexity in Networks. Proceedings of the International Summer School on Complex Systems, Santa Fe Institute, Santa Fé, New Mexico, 2005. disponível em: http://www.santafe.edu/education/csss/ csss05/papers/anastasiadis_et_al._cssssf05.pdf, acesso em Janeiro de 2010.

BAR-YAM, Y. Dynamics of complex systems. Readings, Massachusetts: Addison-Wesley, 1997.

BAUER, R. Gestão da mudança: caos e complexidade nas organizações. S. Paulo: Atlas, 1999.

BERTALANFFY, L. Teoria geral dos sistemas: aplicação à psicologia. In: Anohin, P.; Bertalanffy, L. (org.). Teoria dos sistemas. R. Janeiro: Editora da Fundação Getúlio Vargas, 1976. (série Ciências Sociais).

BERTALANFFY, L. Teoria geral dos sistemas. Petrópolis: Vozes, 1977.

BLECKERB, T.; KERSTEN, W. Complexity Management in Supply Chains: Concepts, Tools and Methods. Berlin: Erich Smith Verlag GmbH, 2006.

CARDOSO, O.; SERRALVO, F. Pluralismo metodológico e transdisciplinaridade na complexidade: uma reflexão para a administração. Revista de Administração Pública, v.43, n.1, p.49-66, 2009.

CHECKLAND, P.; SCHOLES, J. Soft systems methodology in action. Chichester, West Sussex: John Wiley \& Sons, 1999.

CLAUSSEN, J. Offdiagonal Complexity: A computationally quick complexity measure for graphs and network. Physica A. v.375, n.1, p.365-373, 2007.

DEMO, P. Complexidade e aprendizagem: a dinâmica não-linear do conhecimento. S. Paulo: Atlas, 2002.

EISENHARDT, K. Building theories from case study research. Academy of Management Review, v.14, n.4, p.532-550, 1989. 
FLYNN, B.; FLYNN, E. Information-processing alternatives for coping with manufacturing environment complexity. Decision Sciences, v. 30, n 4, p. 10211052, 1999.

GELL-MANN, M. The quark and the jaguar: adventures in the simple and complex. New York: W.H. Freeman and Company, 2000.

GENELOT, D. Mananger dans la complexité: réflexions à l'usage dês dirigentes. Paris: Insep, 2002.

GLEICK, J. Caos: a criação de uma nova ciência. R. Janeiro: Campus, 1990.

HANSEN, P.; OLIVEIRA, L. Proposta de modelo para avaliação sistêmica do desempenho competitivo de arranjos produtivos: o caso do arranjo coureirocalçadista do Vale dos Sinos (RS - Brasil). Produto \& Produção, v.10, n.3, p.61 - 75, 2009.

HEYLIGHEN, F. Building a science of complexity. Proceedings of the 1988 Annual Conference of the Cybernetics Society London, 1988, disponível em http:// pespmc1.vub.ac.be/Papers/BuildingComplexity.pdf, acesso em janeiro/2006.

HOPEMAN, R. Análise de sistemas e gerência de operações. Petrópolis: Vozes, 1977.

JACKSON, M. Systemic methods in management sciences. New York: Plenum Press, 1993.

KALUZA, B.; BLIEM, H.; WINKLER, H. Strategies and metrics for complexity management in supply chains. In T. Bleckerb e W. Kersten (orgs.). Complexity Management in Supply Chains: Concepts, Tools and Methods. Berlin: Erich Smith Verlag GmbH, 2006.

KHISTY, C. e ARSLAN, T. Possibilities of steering the transportation planning process in the face of bounded rationality and unbounded uncertainty. Transportation Research Part C, v.13, p.77-92, 2005.

KIM, J.; WILHELM, T. What is a complex graph? Physica A: Statistical Mechanics and its Applications, v.387, n.11, p.2637-2652, 2008

KLIR, G. Facets of systems sciences. New York: Plenum Press, 1991.

LEWIN, R. Complexidade: a vida no limite do caos. R. Janeiro: Rocco, 1994.

MACIEL, J. Elementos de teoria geral dos sistemas. Petrópolis: Vozes, 1974.
MARKOSE, S. Computability and evolutionary complexity: markets as complex adaptive systems (CAS). The Economic Journal, v.115, n.504, p.159, 2005.

MAXIMIANO, A. Teoria geral da Administração. S. Paulo: Atlas, 1997.

MORIN, E. Ciência com consciência. Rio de Janeiro: Bertrand, 2002.

MORIN, E. Introdução ao pensamento complexo. Porto Alegre: Sulina, 2005.

MORIN, E; LE MOIGNE, J. A inteligência da complexidade. São Paulo: Peirópolis, 2000.

PARKER, D.; STACEY, R. Caos, administração $e$ economia: as implicações do pensamento não-linear. R. Janeiro: Instituto Liberal, 1995

PERONA, M.; MIRAGLIOTTA, G. Complexity management and supply chain performance assessment: A field study and a conceptual framework. International Journal of Production Economics, v.90, n.1, p.103-115, 2004.

PRIGOGINE, I. O fim das certezas: tempo, caos e as leis da natureza. São Paulo: Unesp, 1996.

RIBEIRO, J.; NEWMANN, C. Planejamento e condução de grupos focados. In: Ribeiro, J. (org.) Grupos focados: teoria e aplicações. P. Alegre: FEENGUFRGS-PPGEP, 2003.

ROCHA NETO, I. Gestão de organizações. S. Paulo: Atlas, 2003.

ROESCH, S. Projetos de estágio e de pesquisa em administração: guia para estágios, trabalhos de conclusão, dissertações e estudos de caso. S. Paulo: Atlas, 1999.

RUELLE, D. Acaso e caos. S. Paulo: UNESP, 1993.

SASSI, S. The role of ICT in solving logistic complexity: an economic point of view. In: Bleckerb, T. e Kersten, W. (orgs.). Complexity Management in Supply Chains: Concepts, Tools and Methods. Berlin: Erich Smith Verlag GmbH, 2006.

SELLITTO, M. Medição e controle de desempenho em sistemas de manufatura. Tese de doutorado. Engenharia de Produção. UFRGS, P. Alegre, 2005.

SELLITTO, M.; BORCHARDT, M.; PEREIRA, G. Avaliação numérica da complexidade organizacional de uma cadeia de suprimentos industriais. Anais do XXVII ENEGEP. Foz do Iguaçu: Abepro, 2007. 
SELLITTO, M.; BORCHARDT, M.; PEREIRA, G. Avaliação numérica da complexidade organizacional de um recorte de uma rede pública municipal de serviços de saúde. Anais do XXVIII ENEGEP. Rio de Janeiro: Abepro, 2008a.

SELLITTO, M.; BORCHARDT, M.; PEREIRA, G. Revisão teórica que fundamenta pesquisa sobre a complexidade observada em arranjos e operações interorganizacionais. Produto \& Produção, v.9, n.3, p.67-83, 2008b.

SELLITTO, M.; BORCHARDT, M.; PEREIRA, G.; GUIMARÃES, M. Relative complexity measurement of a supply chain based on information theory. Proceedings of the International Multi-Conference on Complexity, Informatics and Cybernetics: IMCIC 2010. Orlando: International Institute of Informatics and Systemics, 2010.

SIMCHI-LEVI, D.; KAMINSKY, P.; SIMCHI-LEVI, E. Cadeias de suprimentos: projeto e gestão. P. Alegre: Bookman, 2003.

SIMON, H. Architeture of complex systems. In: KLIR, G. (org.) Facets of systems sciences. New York: Plenum Press, 1991.

STERMAN, J. Business dynamics: system thinking and modeling for a complex world. USA: McGrawHill, 2000.

WIENER, N. Cibernética. S. Paulo: Polígono, 1970.

WILHELM, T.; HOLLUNDER, J. Physica A: Statistical Mechanics and its Applications, v.385, n.1, p.385-396, 2007.

YIN, R. Estudo de caso: planejamento e método. P. Alegre: Bookman, 2001.

ZEMAN, J. Significado filosófico da noção de informação. In: Zeman, J.; Goldmann, L.; Granger, G.; Lwoff, A.; Santillana, G.; Frank, H.; Wiener, N.; Bonsack, F. (orgs.). O conceito de informação na ciência contemporânea. S. Paulo: Paz e Terra, 1970. 